\title{
Cesium and bromine doping into hexagonal boron nitride
}

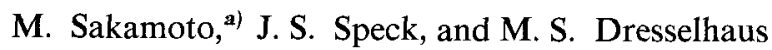 \\ Center for Materials Science and Engineering, Massachusetts Institute of Technology, Cambridge, \\ Massachusetts 02139
}

(Received 19 May 1986; accepted 28 July 1986)

\begin{abstract}
Using a two-zone method, the possible formation of an intercalation compound of hexagonal boron nitride ( $\mathrm{BN}$ ) with $\mathrm{Cs}$ and $\mathrm{Br}_{2}$ was investigated. Only a few percent weight increase was observed by doping $\mathrm{BN}$ with $\mathrm{Cs}$ and $\mathrm{Br}_{2}$. The electron paramagnetic resonance (EPR) signal was significantly modified by $\mathrm{Cs}$ doping, which is attributed to the reaction between the Cs atoms and spin resonance centers ( $\mathrm{N}$ vacancies) in $\mathrm{BN}$; no change in the EPR spectra was observed with $\mathrm{Br}_{2}$ doping. However, the deep blue colored $\mathrm{Cs}-\mathrm{BN}$ complex reported by Mugiya and co-workers was not obtained with the two-zone method. Though no evidence of systematic intercalation reaction in $\mathrm{BN}$ was observed in contrast to graphite host materials, intercalation islands induced by the introduction of Cs atoms were suggested by the transmission electron microscopy (TEM) observations.
\end{abstract}

\section{INTRODUCTION}

Hexagonal boron nitride is a layered hexagonal compound isoelectronic to graphite. Since the early attempts to intercalate $\mathrm{BN}$ by Croft, ${ }^{1}$ there have been several reports regarding various intercalation compounds based on hexagonal boron nitride (BNIC). ${ }^{2-4}$ Except for layer stacking, the structures of graphite and hexagonal boron nitride are similar, as indicated in Table I. For this reason, intercalation compounds based on $\mathrm{BN}$ are expected to be similar to graphite intercalation compounds (GIC's), the most widely studied of the intercalation compounds. The in-plane bond length of $\mathrm{B}-\mathrm{N}$ and the interlayer distance are the same as graphite to within a few percent. ${ }^{5}$ The bonding energies are also similar. These similarities would suggest that intercalation of a dopant into the interlayer space of boron nitride could occur. There is, however, a striking physical difference between these materials. While graphite is a semimetal with a small band overlap, boron nitride is a transparent insulator with a band gap of $\sim 5 \mathrm{eV} .^{6-10}$ The wide band gap character of $\mathrm{BN}$ greatly reduces the intercalation reaction because the electronic system is very stable and inactive due to the wide band gap. Activation of electrons across this wide gap is required for a chemical reaction to occur.

The simple physicochemical considerations listed above have all been confirmed experimentally, while both positive and negative results on the intercalation of BN have been reported. ${ }^{2,3}$

Using black lustrous BN powder, $\mathrm{Croft}^{1}$ reported a weight increase ranging from $2 \%-13 \%$ by the reaction with $\mathrm{FeCl}_{3}, \mathrm{AlCl}_{3}$, and $\mathrm{NH}_{3}$. He also observed an exfoliation, one of the characteristics of the intercalation

\footnotetext{
a) Present address: Toshiba R \& D Center, Komukai Saiwai-ku, Kawasaki 210, Japan.
}

process, of a resultant complex of the fine powder. Rüdorff and Stumpp, ${ }^{2}$ however, found no change in weight and color of their white $\mathrm{BN}$ powder by reactions similar to those employed by Croft. ${ }^{1}$

Freeman and Larkindale ${ }^{11,12}$ examined both acceptor (metal halides) and donor ( $\mathrm{Li}, \mathrm{Na}, \mathrm{K})$ doping into a white $\mathrm{BN}$ powder. They detected an expansion of the interlayer distance in $\mathrm{FeCl}_{3}$-doped $\mathrm{BN}$ and for the donors they observed a weight increase corresponding to the formula $(\mathrm{BN})_{13} \mathrm{~K}$ together with an expansion of the interlayer distance to $4.4 \AA$.

Mugiya et al. ${ }^{13}$ also examined alkali metal doping of $\mathrm{BN}$ and reported a deep blue complex of $\mathrm{BN}$ with $\mathrm{Cs}$. Ohhashi and Shinjo ${ }^{14}$ intensively studied $\mathrm{FeCl}_{3}$-doped BN and found no appreciable absorption in the Mössbauer spectrum, which indicated that the $\mathrm{Fe}$ content of the $\mathrm{FeCl}_{3}$-doped $\mathrm{BN}$ powder was extremely small. They inspected the reaction conditions and concluded that the pink color and $\mathrm{x}$-ray diffraction peaks reported ${ }^{11,12}$ as characteristics of a $\mathrm{BN}$ intercalation compound with $\mathrm{FeCl}_{3}$ should be attributed to $\mathrm{FeOCl}$ formed on the surface of the $\mathrm{BN}$ and associated with a slight addition of $\mathrm{H}_{2} \mathrm{O}$ in the reaction tubes.

Bartlett et al. ${ }^{15}$ reported a very unstable, deep blue

TABLE I. Properties of graphite and boron nitride BN.

\begin{tabular}{lccccc}
\hline \hline & $\alpha^{\mathrm{a}}$ & $a_{0} / \sqrt{3}(\AA)^{\mathrm{b}}$ & $c_{0} / 2(\AA)^{\mathrm{c}} E_{g}(\mathrm{eV})^{\mathrm{d}} \sigma(\mathrm{S} / \mathrm{cm})^{\mathrm{e}}$ \\
\hline Graphite & $120^{\circ}$ & 1.42 & 3.35 & semimetal & $2.5 \times 10^{4}$ \\
BN & $120^{\circ}$ & 1.45 & 3.33 & $4 \sim 5 \mathrm{eV}$ & $\sim 0$ \\
& & & & & \\
\hline \hline
\end{tabular}

${ }^{a} \alpha$ denotes the bond angle.

${ }^{\mathrm{b}} a_{0} / \sqrt{3}$ is the nearest-neighbor in-plane distance.

${ }^{c} c_{0} / 2$ is the interplanar distance.

${ }^{d} E_{\mathrm{g}}$ is the band gap.

e $\sigma$ is the conductivity. 
colored complex having a nominal formula (BN) ${ }_{4} \mathrm{SO}_{3} \mathrm{~F}$ with layer distance of $8.02 \AA$ formed by the reaction of $\mathrm{BN}$ with $\left(\mathrm{SO}_{3} \mathrm{~F}\right)_{2}$. Hooley, ${ }^{3}$ however, observed only a small weight increase $(0.1 \% \sim 0.6 \%)$ with $\left(\mathrm{SO}_{3} \mathrm{~F}\right)_{2}$ doping.

Some of the early attempts at intercalation were obtained using black $\mathrm{BN}$ polycrystalline powder that contained high concentrations of impurities like carbon and exhibited very large deviations from stoichiometry in the $\mathrm{B}: \mathrm{N}$ ratio (naturally occurring $\mathrm{BN}$ sometimes contains more than $10 \%$ of $\mathrm{MgO}$ ) ${ }^{16}$ Thus the discrepancy of the results for the intercalation of $\mathrm{BN}$ can be explained to some extent by sample variability.

Recently high-purity pyrolytic BN samples have become available as source materials for fine ceramics. This material allows us to have better control of the host material and gives more reproducible results on the intercalation of $\mathrm{BN}$. In this paper we describe the effect of doping high-purity $\mathrm{BN}$ powder and flakes with cesium metal and bromine gas. We monitor the extent of intercalation by electron paramagnetic resonance (EPR) and transmission electron microscopy (TEM). Vacancies in $\mathrm{BN}$ can be analyzed very sensitively (down to 0.5 ppm deviation from stoichiometry) by EPR ${ }^{17}$; in fact, a small amount of carbon contamination from the furnace wall was detected ${ }^{18}$ during the heating process. However, there has been no previous report, to our knowledge, of the EPR technique applied to detect the intercalation of a BN compound. The TEM observations were used to provide detailed information about local structural changes on a scale too small to affect the $\mathrm{x}$-ray diffraction pattern significantly. Here $\mathrm{Cs}$ and $\mathrm{Br}_{2}$ are selected as possible intercalants because they are common elemental dopants, which in $\mathrm{BN}$ are expected to be donors and acceptors, respectively. They both have a high vapor pressure at relatively low temperature, and thus eliminate the complications of dopant decomposition that are inherent to molecular dopants (e.g., $\mathrm{AsF}_{5}$ ) and also of thermal annealing effects of the host materials. Moreover, their heavy masses make detection easier in weight increase measurements.

\section{EXPERIMENTAL}

Four BN samples from different sources were used in the studies reported in this paper. They are

(i) sample A: white powder (Fluka Chemical Corporation, nominal purity $99 \%$ ),

(ii) sample B: white powder (Denkikagaku Kogyo, nominal purity $99 \%$ ),

(iii) sample C: white powder (Pfaltz and Bauer, Inc., nominal purity $97 \%$ ),

(iv) sample D: highly oriented BN (HOBN) transparent flake (Union Carbide Corp.), having a preferred orientation of the $\mathrm{c}$ axis perpendicular to the flake surface with a mosaic spread of less than $5^{\circ} .^{19}$
Prior to doping or intercalation, sample $\mathrm{D}$ showed a tannish yellow color that might be ascribed to either a carbon impurity or a nitrogen vacancy. In fact, Fourier transform infrared (FTIR) transmission spectra (in the range from $1000-4000 \mathrm{~cm}^{-1}$ ) of sample $D$, showed in addition to the strong $1375 \mathrm{~cm}^{-1}$ absorption peak characteristic of hexagonal $\mathbf{B N},{ }^{20}$ weak absorption peaks at 2550 and $2300 \mathrm{~cm}^{-1}$. On the other hand, the IR spectra of samples A, B, C were indistinguishable to within experimental error and showed only the $1375 \mathrm{~cm}^{-1}$ peak.

Chemical doping of $\mathrm{BN}$ was carried out using the two-zone method similar to the synthesis of GIC's. This method is advantageous because no condensation of excess dopant onto the BN surface is expected due to the temperature gradient. By employing the two-zone method we could avoid washing the doped BN, which could damage unstable compounds. The BN samples were put into the sample compartment of the two-zone tube that contained a Cs source separated by a breakable seal as shown in Fig. 1. The whole system was pumped down to a pressure of less than $10^{-5}$ Torr and baked at about $250^{\circ} \mathrm{C}$ for $20 \mathrm{~min}$ to degas the tube walls and the samples. After baking, the breakable seal was opened and the Cs metal (99.999\% purity) was distilled and transferred in vacuum into the dopant compartment of the two-zone tube. After the two-zone tube was sealed off and cut away from the vacuum line and Cs source, it was kept in the two-zone configuration for twenty days, where the temperature of the dopant and $\mathrm{BN}$ samples were 220 and $250^{\circ} \mathrm{C}$, respectively.

For bromine doping, an $\mathrm{L}$-shaped tube was used as shown in Fig. 2. The vacuum-dried BN samples were placed in the sample finger, and bromine was poured into the dopant finger inside an argon-filled glove bag. The evacuation valve was closed and the bromine was

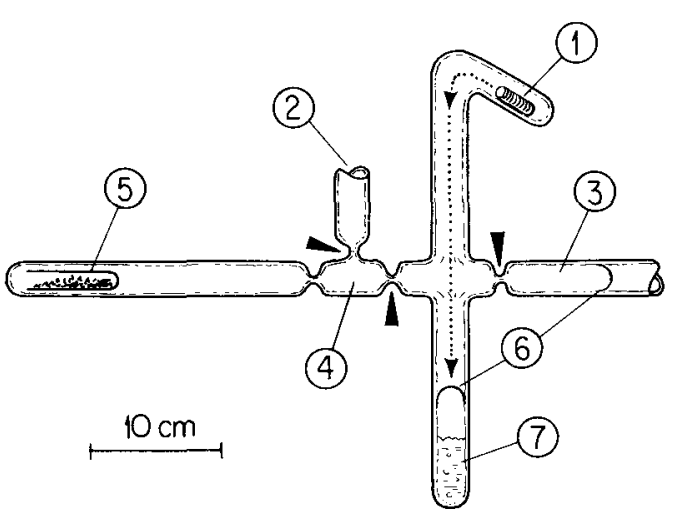

FIG. 1. Arrangement of the two-zone reaction tube for Cs doping of BN. The diagram is labeled as follows: (1) a glass coated hammer, (2) to be joined to the vacuum system, (3) the Cs recess, (4) the Cs dopant compartment, (5) the BN sample, (6) breakable seal, (7) a Cs source. The tube diameter is $12 \mathrm{~mm}$. The glass necks indicated by solid triangles are sealed off at appropriate steps in the preparation process. 


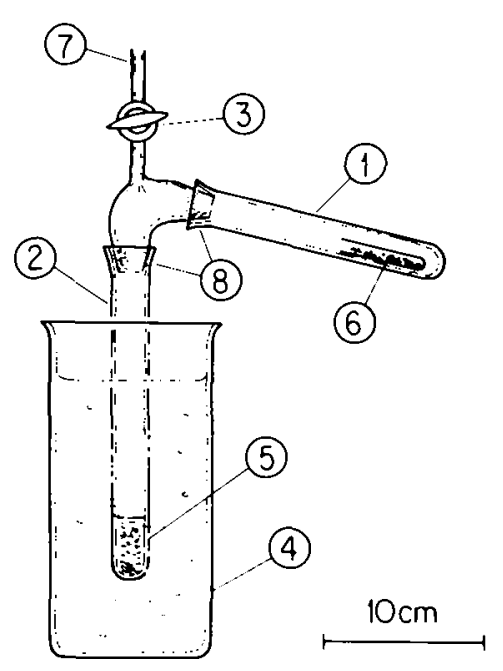

FIG. 2. Arrangement of the reaction tube for $\mathrm{Br}_{2}$ doping of $\mathrm{BN}$. The diagram is labeled as follows: (1) a sample finger, (2) a dopant finger, (3) an evacuation valve, (4) a temperature-controlled water bath, (5) $\mathrm{Br}_{2}$ reservoir, (6) $\mathrm{BN}$ sample, (7) to be joined to the vacuum system, (8) vacuum tight joints.

solidified by cooling the dopant finger with liquid nitrogen. Then the tube was evacuated through an evacuation valve and sealed. For the attempted intercalation the dopant finger was dipped into a cooled bath with a temperature of $8{ }^{\circ} \mathrm{C}$, and the sample finger was kept at $20^{\circ} \mathrm{C}$ for 15 days, after which the tubes were opened in a glove bag that had been thoroughly flushed with pure dry argon gas. The samples were then sealed into airtight containers and EPR tubes for weighing and for EPR measurements. Powder $x$-ray diffraction measurements were carried out with the samples sealed between thin $(0.15 \mathrm{~mm}$ thick) glass plates. The pristine and Cs-doped BN samples $\mathrm{D}$ were examined by TEM.

\section{RESULTS AND DISCUSSION}

By Cs doping, all the white powder BN samples became slightly bluish gray. Several dark spots with 0.1-1 mm diameter appeared in the flakes $(5 \times 5 \times 0.05$ $\mathrm{mm}$ ) of sample $\mathrm{D}$. The bluish-gray color of the powder samples was rapidly bleached to white gray by exposure to air. The color faded slowly even in an argon atmosphere. No color change was observed by just heating the samples in vacuum at $250^{\circ} \mathrm{C}$.

Upon bromine doping, the white powder BN samples became somewhat ivory colored, especially sample $C$, which became even more deeply colored (a light $\tan )$. The bromine-doped sample A bleached to white by leaving it in an argon atmosphere for 2 days. Only slight fading was observed in samples $B$ and $C$, and no change in appearance was detected in sample $D$ after 2 days in an argon atmosphere.

The weight increases of the powder samples mea- sured immediately after opening the reaction tubes are listed in Table II. A small weight increase in Cs-doping $\mathrm{BN}$ corresponding to the formula $(\mathrm{BN})_{300} \mathrm{Cs}$ was observed in samples $B$ and $C$. The detected weight increases in the bromine-doped samples corresponded to the formula $(\mathrm{BN})_{160} \mathrm{Br}$. The $\mathrm{x}$-ray diffraction patterns of the Cs-doped powders ( A and B) showed essentially no difference from their pristine state.

The characteristics of the EPR spectra of the pristine samples from $A$ to $D$ are summarized in Fig. 3 and Table III. Only a narrow single line signal with $\Delta H$ of 9 $G$ was detected in sample A [see Fig. 3(a) ]. In constrast, sample B yielded a typical ten-line signal attributed to unpaired electrons trapped by isolated single nitrogen vacancies, ${ }^{21}$ which interact with threc equivalent ${ }^{11} B$ nuclei with a spin of $3 / 2$. The linewidth of the tenline spectrum determined for the peak of the envelope of the ten peak spectrum was $27 \mathrm{G}$ and showed good agreement with the values previously reported. ${ }^{17.18,21,22}$ In sample $C$ this ten-line spectrum was observed to be superimposed on a large broad signal (with a linewidth $\Delta H$ as large as $500 \mathrm{G}$ ) as shown in Fig. $3(\mathrm{c})$, where the sharp signal at the center is presented on an expanded scale in Fig. 3(d) to show the characteristic spectrum with features similar to trace 3 (b). Sample D showed a signal similar to that of sample C, i.e., a superposition of a ten-line spectrum with a broad background signal as shown in Fig. 3(e) and Fig. 3(f) for $\theta=0$ and $\theta=90^{\circ}$, respectively, where $\theta$ is an angle between the $c$ axis and the magnetic field. As seen by comparing Figs. 3(e) and 3 (f), the ten-line spectrum in sample $D$ is strongest at $\theta=90^{\circ}$ and is almost undetected at $\theta=0$. The linewidth $\Delta H$ of the broad component in sample $\mathrm{D}$ ranges from $100-200 \mathrm{G}$, depending on the angle $\theta$, and is less than half of the linewidth of the broad signal in sample C. The broad spectrum in both samples shows a Gaussian line shape associated with an inhomogeneously broadened line.

The difference in magnitude of the anisotropy of the $g$ factor of the ten-line spectrum and the broad signal in sample $D$ is emphasized in Fig. 4. The angular dependence of the $g$ factor of the ten-line signal (plotted as $g_{10}$

TABLE II. Weight increase $\Delta w$ of $\mathrm{BN}$ samples by $\mathrm{Cs}$ and $\mathrm{Br}_{2}$ doping

\begin{tabular}{ccc}
\hline \hline Sample & Dopant & $\Delta w[\%]^{3}$ \\
\hline A & $\mathrm{Cs}$ & $\sim 0$ \\
$\mathrm{~B}$ & $\mathrm{Cs}$ & 2 \\
$\mathrm{C}$ & $\mathrm{Cs}$ & 2 \\
$\mathrm{~A}$ & $\mathrm{Br}_{2}$ & 2 \\
$\mathrm{~B}$ & $\mathrm{Br}_{2}$ & 2 \\
$\mathrm{C}$ & $\mathrm{Br}_{2}$ & 2 \\
\hline
\end{tabular}

${ }^{a}$ Experimental error is $\pm 0.3 \%$. 


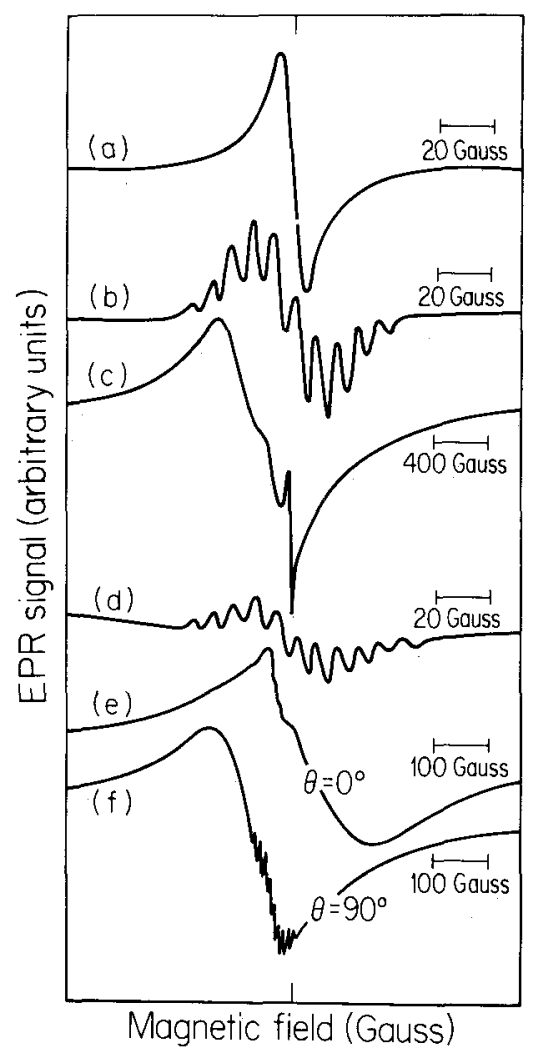

FIG. 3. The EPR signal traces of the pristine $B N$ samples. The diagram is labeled as follows: (a) sample A, (b) sample B, (c) sample C (wide scan trace), (d) sample $C$ (expanded trace of the signal in $c$ ), (e) sample $\mathrm{D}$ at $\theta=0^{\circ}$, (f) sample $\mathrm{D}$ at $\theta=90^{\circ}$, where $\theta$ denotes the angle between the magnetic field and the $c$ axis of sample $D$. The magnetic field at the center of the figures is set equal for traces (a)(d) and for traces (e) and (f).

in Fig. 4) is similar to that reported by Moore and Singer, ${ }^{18}$ which could be represented by the relation

$$
g=g_{1}+\Delta g \cos ^{2} \theta,
$$

where $g_{\perp}\left(g\right.$ value at $\left.\theta=90^{\circ}\right)$ and $\Delta g$ are given by 2.0042 and $-1.4 \times 10^{-3}$, respectively, in our experiments, and these values are fairly close to the values of $g_{1}=2.0032$ and $\Delta g=-9.5 \times 10^{-4}$ reported by Moore and Singer. ${ }^{18}$ The angular dependence of the $g$ factor for the broad signal $g_{B}$ is discussed below. The linewidth $\Delta H$ and the intensity (the product of $\Delta H$ and line height) $I$ of the broad signal in sample $\mathrm{D}$ relative to the $\theta=0$ value are plotted in Fig. 5 as a function of the direction of the magnetic field. Both $\Delta H$ and $I$ show an angular dependence different from each other and from a $\cos ^{2} \theta$ dependence. The $g$ values are all between 2.004 and 2.006 with the exception of the broad signals in samples $\mathrm{C}$ and $\mathrm{D}$.

With Cs doping, the EPR signal of samples B and C changed considerably, as summarized in Table III. The ten-line signal of the BN sample B was attenuated upon Cs doping as shown in Fig. 6(a) and accompanied by a narrowing of the linewidth to $13 \mathrm{G}$, about half of the pristine value [see Fig. 3(b) for comparison]. Both the ten-line signal and the broad signal of sample $\mathrm{C}$ [see Figs. 3(c) and 3(d)] disappeared with Cs doping, and simultaneously a weak narrow single line signal appeared as shown in Fig. 6(b) and in an expanded form in Fig. 6(c). However, the narrow single line signal in sample A showed no change before and after Cs doping. Though no clear EPR spectra of the Cs-doped BN sample $\mathrm{D}$ was detected due to the small quantities of the doped sample, a change similar to that of sample $\mathrm{C}$ could be expected.

A thermal annealing effect of the ten-line signal has been reported by Katzir et al., ${ }^{23,24}$ and based on their work, the thermal annealing effect was expected to be small under our doping conditions. Further evidence in support of this point is provided by the thermal annealing results for sample $\mathrm{C}$, which showed almost no change in the EPR spectrum. Therefore we attribute the spectral change upon doping to the effects of Cs doping. As shown in Table III, the $g$ values of all the Cs-doped samples were almost the same.

It was suggested by Moore and Singer ${ }^{18}$ that the narrow single line signal arises from a dispersed carbon phase in or on the BN powder; this provides a consistent explanation for the narrow signal observed in the pristine sample A and the residual signal in Cs-doped samples $\mathrm{B}$ and $\mathrm{C}$. The small amount of carbon-dispersed phase will behave differently from pure graphite and will not necessarily be affected by $\mathrm{Cs}$ doping. On the other hand, nitrogen vacancies that give the ten-line signal have bound unshared electrons (like color centers) whose energy levels were calculated to be $1.4 \mathrm{eV}$ below the conduction band edge $(7.9 \sim 8.7 \mathrm{eV}$ below the vacuum level) by Zunger and Katzir. ${ }^{25,26}$ Though the simple energy level argument cannot fully explain the intercalation reaction even between alkali metals and graphite, an electron transfer reaction between the Cs atom and a defect (nitrogen vacancy) energy level which appears in the energy gap of BN would be much easier than that between a $\mathrm{Cs}$ atom and conduction band state in $\mathrm{BN}$. We therefore attribute the disappearance of the ten-line spectrum upon Cs doping to a binding of the Cs atom to the nitrogen vacancy.

The broad signal observed in samples C and D in their pristine state has not been previously reported in detail. The results obtained thus far suggest a strong sample dependence of this EPR signal, which might be due to the sample atomization or heating history. The line shape for samples C and D was closer to Gaussian than for sample A. This near-Gaussian EPR line shape may be due to inhomogeneous broadening similar to that observed in the EPR spectra of color centers in alkali halides. ${ }^{27}$ In fact, the linewidth of the broad signal in sample D (highly oriented BN) is narrower than that 
TABLE III. EPR characteristics of the pristine and doped BN samples.

\begin{tabular}{|c|c|c|c|c|}
\hline Sample & EPR signal & $\Delta H$ (Gauss) & {$[S]\left(\mathrm{cm}^{3}\right)^{\mathrm{a}}$} & $g$ \\
\hline \multicolumn{5}{|l|}{ undoped } \\
\hline A & single & 9 & $5.4 \times 10^{16}$ & 2.005 \\
\hline $\mathrm{B}$ & ten lines & 27 & $9.6 \times 10^{17}$ & 2.004 \\
\hline \multirow[t]{2}{*}{$\mathrm{C}$} & ten lines & 30 & $2.4 \times 10^{17}$ & 2.004 \\
\hline & + broad & 450 & $1.2 \times 10^{19}$ & $2.13 \sim 2.21$ \\
\hline \multirow[t]{2}{*}{$\mathrm{D}$} & ton lines & 27 & $3.0 \times 10^{17^{n}}$ & $\sim 2.004$ \\
\hline & + broad & $100 \sim 200^{\circ}$ & $7.0 \times 10^{14^{\prime \prime}}$ & $1.97 \sim 2.03^{\mathrm{c}}$ \\
\hline \multicolumn{5}{|l|}{ Cs doped } \\
\hline A & single & 9 & $6.0 \times 10^{16}$ & 2.005 \\
\hline \multirow[t]{2}{*}{ B } & single & 13 & $1.0 \times 10^{18}$ & 2.006 \\
\hline & (with weak ten lines) & & & \\
\hline $\mathrm{C}$ & single & 18 & $1.0 \times 10^{18}$ & 2.005 \\
\hline \multicolumn{5}{|c|}{$\mathrm{Br}_{2}$ doped } \\
\hline A & single & 10 & $7.0 \times 10^{16}$ & 2.004 \\
\hline $\mathrm{B}$ & ten lines & 28 & $1.5 \times 10^{18}$ & 2.004 \\
\hline \multirow[t]{2}{*}{$\mathrm{C}$} & ten lines & 20 & $2.5 \times 10^{17}$ & 2.004 \\
\hline & + broad & 450 & $1.2 \times 10^{19}$ & 2.17 \\
\hline \multicolumn{5}{|c|}{ Heat annealed ${ }^{c}$} \\
\hline \multirow[t]{2}{*}{$\mathrm{C}$} & ten lines & 25 & $2.2 \times 10^{17}$ & 2.004 \\
\hline & + broad & 600 & $1.2 \times 10^{19}$ & 2.18 \\
\hline
\end{tabular}

${ }^{a}$ The spin density $[S]$ responsible for each signal is estimated from the integrated area of the EPR signal using a standard sample.

'Value at $\theta=90^{\circ}$ where $\theta$ is the angle between the $c$ axis and magnetic field.

'Depending on orientation.

¿Value at $\theta=0^{\circ}$.

"At $250^{\circ} \mathrm{C}$ for 20 days in vacuum.

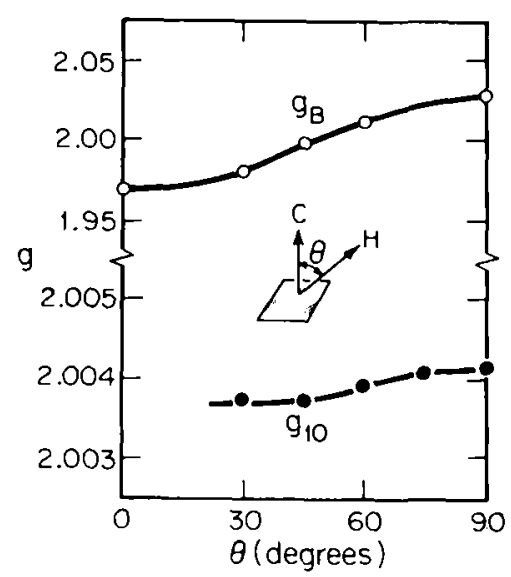

FIG. 4. Anisotropy of the $g$ factors of the broad signal $\left(g_{B}\right)$ and the ten-line signal $\left(g_{10}\right)$ for sample $D$. The angle between the magnetic field and $\mathrm{c}$ axis is denoted by $\theta$. The ten-line signal became obscured for small $\theta$ so that $g_{10}$ could not be determined accurately for small $\theta$.

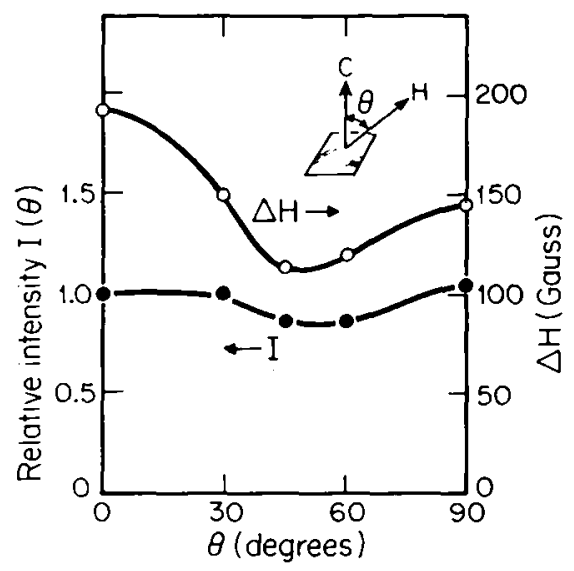

FIG. 5. Anisotropy of the relative intensity $I(\theta)$ and the linewidth (peak-to-peak width) $\Delta H$ of the broad EPR signal of the pristine sample D. $I(\theta)$ is the product of the peak-to-peak height and $\Delta H$, normalized to the value at $\theta=0^{\circ}$, where $\theta$ denotes the angle between the magnetic field and $\mathrm{c}$ axis. 


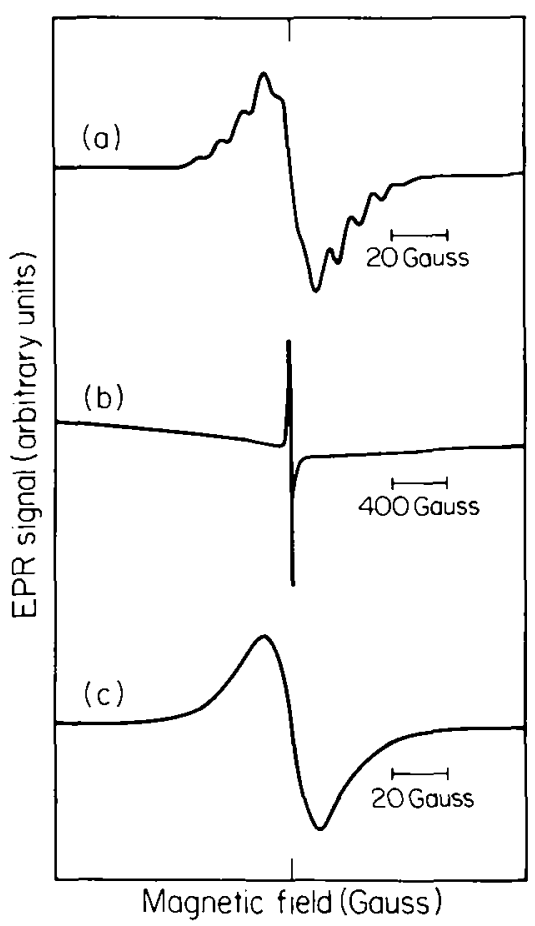

FIG. 6. EPR signal traces of the Cs-doped sample B,(a), and sample $\mathrm{C},(\mathrm{b})$. The sharp line in (b) is shown on an expanded scale in (c).

in sample $C$ (fine powder), consistent with the difference in structural order between the samples. Although the identity of the defect responsible for the broad resonance line is still uncertain, it seems plausible to identify it with a variety of clusters of nitrogen vacancies and disrupted bonds, which extend both along the $\mathrm{c}$-axis and in-plane directions of the layered hexagonal BN. In fact, the anisotropy of the linewidth and the intensity of the broad signal do not exhibit a $\cos ^{2} \theta$ dependence, as shown in Fig. 5, while the $g$ factor shows a $\cos ^{2} \theta$ dependence, as shown in the plot of $g_{B}$ vs $\theta$ in Fig. 4. This suggests that the defects responsible for the broad EPR signal maintain an in-plane character in the layered material. On the other hand, the intensity of the ten-line signal decreases monotonically with $\theta$ as $\theta \rightarrow 0^{\circ}$, and almost disappears at $0^{\circ}$. This behavior, as well as the $\cos ^{2} \theta$ dependence of $g$ factor [see Eq. (1)], are both a manifestation of the strong anisotropy of the defects.

The spin concentrations estimated from the EPR intensity are indicated in Table III for each signal. The high spin concentration of $10^{19} \mathrm{~cm}{ }^{3}$ responsible for the broad EPR signal in the pristine sample $C$ represents one spin per 6000 of $(B N)$ pairs. The spin concentration of $10^{18} \mathrm{~cm}^{-3}$ corresponding to the single line spectrum observed in the Cs-doped sample $\mathrm{C}$ was slightly higher than that for the ten-line signal in the pristine state. This suggests that the single peak in the Cs-doped sample C was generated through some conversion process of the vacancies, which gave both the ten-line spectrum and the broad signal to clusters of carbon atoms. It has been pointed out ${ }^{18,21,23-25}$ that the nitrogen vacancies are often accompanied by carbon impurity atoms.

The spin concentration values associated with the EPR signals that disappeared with Cs doping were almost the same order of magnitude as the Cs atomic concentration calculated from the measured weight increases, consistent with identification of the disappearance of the ten-line spectrum with a binding of the $C s$ atom to a nitrogen vacancy.

In contrast to the Cs doping, the bromine doping made no qualitative change in the EPR spectra of any of the powder samples, as summarized in Table III. The weight increase detected in bromine-doped samples could thus be ascribed for the most part to the physisorption on the powder particles. The spin density responsible for the broad EPR spectrum in $\mathrm{Br}_{2}$-doped sample $\mathrm{C}$, however, decreased by one order of magnitude from the pristine value, which implies that some of the defects reacted with bromine. The deep ivory color of the bromine-doped sample $\mathrm{C}$ might be attributed to the strong absorption due to a chemical reaction between $\mathrm{Br}_{2}$ molecules and defects in the $\mathrm{BN}$ sample. Neither the ten-line nor the narrow single line EPR spectra were affected significantly by bromine doping.

Examination of doped and pristine samples by transmission electron microscopy revealed that the Csdoped samples contained a density of $\sim 10^{15} \mathrm{~cm}^{-3}$ small circular defects ranging in size from $100-800 \AA$ as shown in Fig. 7. No such defect contrast was observed in the pristine samples. In Fig. 7 a two beam diffracting condition was established with $\mathbf{g}=(102)$, where $g$ is the reciprocal lattice vector for the excited Bragg reflection in a two beam condition. From the dynamical theory of electron diffraction, ${ }^{28}$ it is known that the visibility of a defect requires $\mathbf{g} \cdot \mathbf{R} \neq 0$, where $\mathbf{R}$ is the displacement field for the defect. The displacement field for an extrinsic prismatic dislocation loop is given $\mathrm{as}^{28}$ :

$$
\begin{aligned}
\mathbf{R}= & \frac{1}{2 \pi}\left[\mathbf{b} \phi+\mathbf{b} \frac{\sin 2 \phi}{4(1-v)}+(\mathbf{b} \times \mathbf{u})\right. \\
& \left.\times\left(\frac{1-2 v}{2(1-v)} \ln r+\frac{\cos 2 \phi}{4(1-v)}\right)\right],
\end{aligned}
$$

where $\mathbf{b}$ is the Burgers vector for the loop, $\phi$ is a geometrical factor dependent on specimen geometry, $v$ is Poisson's ratio, $r$ is the distance from the dislocation core, and $\mathbf{u}$ is a unit vector tangent to the dislocation line. Generally, the (b×u) term in Eq. (2) is small and can be neglected to first order, leading to the well-known $\mathbf{g} \cdot \mathbf{b} \neq 0$ criterion for defect visibility. The circular defects shown in Fig. 7 were invisible when only $(h k 0)$ reflections were excited, thus confirming the dislocation loop identification. The TEM data are entirely consistent with images of extrinsic prismatic dislocation loops with $\mathbf{b}=(c / 2)[001]$; the additional layer of material bound- 


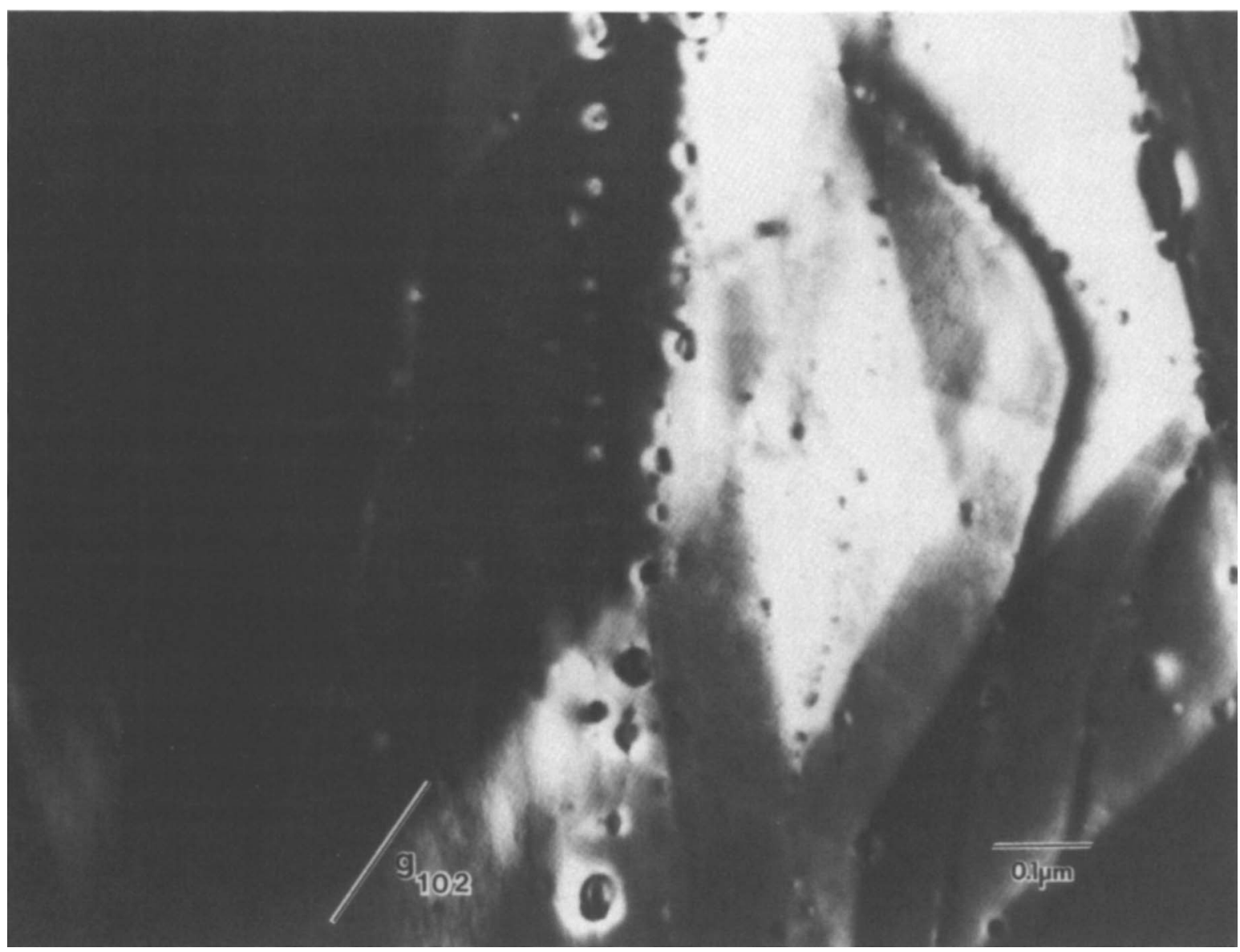

FIG. 7. A TEM of a Cs-doped BN sample exhibiting prismatic dislocation loops. No such dislocation loops were observed prior to Cs doping.

ed by the loop is Cs from the doping reaction. Individual prismatic dislocation loops represent, in this case, small intercalation islands that are precursors to the formation of a staged intercalation compound. Assuming an average loop diameter of $\sim 400 \AA$ and thickness of $3 \AA$, the volume fraction of material in the loop is $\sim 0.1 \%$. Within the limits of the approximation of loop density and thickness, this result compares favorably with the weight uptake measurements of $1 \%-3 \%$, which was measured in samples B and C (see Table II).

There is faint contrast between the loops shown in Fig. 7, which leads to the speculation that the loops are nucleated heterogeneously on glissile $\mathrm{BN}$ dislocations of character $\mathbf{b}=[m n 0]$. There are several reasons that dopant precipitation at $\mathrm{BN}$ dislocations is anticipated: (1) A high local concentration of dopant exists along the dislocation cores due to pipe diffusion. (2) The energy of the BN crystal can be decreased by reduction of dislocation core volume through precipitation of Cs. (3) The bond energy of the BN crystal can be reduced by migration of Cs to dangling bonds which exist at dislocation cores. These conjectures are consistent with the changes in the EPR signal caused by Cs doping.
The Raman scattering from the transparent portion of the Cs-doped sample D showed only a peak at $\sim 1370$ $\mathrm{cm}^{-1}$, which is characteristic of pristine $\mathrm{BN} .{ }^{29-31}$ Not even a peak at $\sim 1370 \mathrm{~cm}^{-1}$ was observed in the Raman scattering from the dark spots on the Cs-doped sample $\mathrm{D}$, which suggests that the dark spots were not due to a modified form of BN. Moreover, since the $1370 \mathrm{~cm}^{-1}$ Raman peak is due to an intralayer mode, it would not necessarily be sensitive to a change in interlayer distance by Cs insertion into a defect region as long as the force constants of the $\mathrm{BN}$ bonds were not changed by a charge transfer reaction. These may be the reasons why no signal other than the pristine peak was observed in the Raman spectrum for the Cs-doped samples.

Mugiya and co-workers ${ }^{13}$ reported the formation of a deep blue Cs-BN upon Cs doping of the white BN powder. Dark spots appearing on the Cs-doped sample $\mathrm{D}$, seem, from their color, to be similar to this Cs-BN complex. However, the Cs concentration in the dark spots could not be detected by electron probe microanalysis: however, there is the possibility that the dark spots were not on the surface but located beneath the BN layer and thus were not sensitive to analysis by the electron 
microprobe. The reaction method of Mugiya et al. ${ }^{13}$ was not a two-zone method as was used in this work, but rather involved direct contact with the molten Cs metal and $\mathrm{BN}$ powder at $200^{\circ} \mathrm{C}$. Thus more complicated reactions like surface adsorption would be expected in their samples as well as a faster reaction rate than by the twozone method, which is basically a vapor transport method. This might be the reason for the different appearance of the BN-doped samples between the experiments of Mugiya et al. ${ }^{13}$ and the experiments reported here.

In summary, the EPR results suggest that Cs doping of $\mathrm{BN}$ proceeds through defects in the $\mathrm{BN}$ host, consistent with doping, rather than intercalation. The TEM results, however, suggest local coalescence of the Cs into "intercalation islands," thereby giving rise to a local expansion of the interlayer distance. Thus the addition of Cs to BN observed in this work cannot be regarded as a systematic intercalation process. We further find no significant doping of BN by bromine. We note, however, that recently Tanuma and $\mathrm{Okabe}^{32}$ have successfully prepared stage 4 or $5 \mathrm{BiCl}_{3}$ intercalation compounds in BN. This leads to the possibility of an exciting new class of staged intercalation compounds with an insulator as the host.

\section{ACKNOWLEDGMENTS}

The authors are grateful to Dr. G. Dresselhaus, Dr. T. Enoki, and Dr. G. Braunstein for valuable discussions and comments, and to Dr. A. W. Moore for providing the compression-annealed highly oriented BN used in this work as the sample D.

Financial support from NSF-MRL Grant No. DMR-84-18718 is gratefully acknowledged.

\section{REFERENCES}

${ }^{1}$ R. C. Croft, Aust. J. Chem. 9, 206, (1956).

${ }^{2}$ W. Rüdorff and E. Stumpp, Z. Naturforsch. Teil B 13, 459 (1958).

${ }^{3}$ J. G. Hooley, Carbon 21, 181 (1983).
${ }^{4} \mathrm{~S}$. Tanuma (private communication).

${ }^{5} \mathrm{~J}$. G. Hooley, in Preparation and Crystal Growth of Materials with Layered Structures, edited by R. M. A. Leith (Reidel, Dordrecht, Holland, 1978).

${ }^{6}$ A. Balzarotti and M. Grandolfo, Phys. Rev. Lett. 20, 9 (1968).

${ }^{7}$ D. L. Greenaway, G. Harbeke, F. Bassani, and E. Tosatti, Phys. Rev. 178, 1340 (1969).

${ }^{8}$ G. S. Painter and D. E. Ellis, Phys. Rev. B 1, 4747 (1970).

${ }^{9}$ F. Bassani and G. P. Parravicini, Nuovo Cimento B 50, 95 (1967).

${ }^{10}$ J. Zupan, Phys. Rev. B 6, 2477 (1972).

${ }^{11}$ A. G. Freeman and J. P. Larkindale, J. Chem. Soc. A 7, 1307 (1969).

${ }^{12}$ A. G. Freeman and J. P. Larkindale, Inorg. Nucl. Chem. Lett. 5, 937 (1969).

${ }^{13}$ C. Mugiya, N. Ohigashi, Y. Mori, and H. Inokuchi, Bull. Chem. Soc. Jpn. 43, 287 (1970).

${ }^{14} \mathrm{~K}$. Ohhashi and T. Shinjo, Bull. Inst. Chem. Res. Kyoto Univ. 55, 441 (1977).

${ }^{15}$ N. Bartlett, R. N. Biagioni, B. W. McQuillan, A. S. Robertson, and A. C. Thompson, J. Chem. Soc. Chem. Comm. 1978, 200.

${ }^{16}$ G. R. Finley and G. H. Fetterley, Ceram. Bull. 31, 141 (1952).

${ }^{17}$ M. B. Khusidman and V. S. Neshpor, Sov. Phys.-Solid State 10, 975 (1968).

${ }^{18}$ A. W. Moore and L. S. Singer, J. Phys. Chem. Solids 33, 343 (1972).

${ }^{19}$ A. W. Moore, Nature 221, 1133 (1969).

${ }^{20}$ E. G. Brame, J. L. Margrave, and V. W. Meloche, J. Inorg. Nucl. Chem. 5, 48 (1957).

${ }^{21}$ D. Geist, in Boron and Refractory Borides, edited by V. I. Matkovish (Springer, Berlin, 1977).

${ }^{22}$ D. Geist and G. Römelt, Solid State Commun. 2, 149 (1964).

${ }^{23}$ A. Katzir, J. T. Suss, and A. Halperin, Phys. Lett. A 41, 117 (1972).

${ }^{24}$ A. Katzir, J. T. Suss, A. Zunger, and A. Halperin, Phys. Rev. B 11, 2370 (1975).

${ }^{25}$ A. Zunger and A. Katzir, Phys. Rev. B 11, 2378 (1975).

${ }^{26}$ A. Zunger, J. Chem. Phys. 62, 1861 (1975).

${ }^{27}$ J. J. Markham, in Solid State Physics, Supplement 8, edited by F. Seitz and D. Turnbull (Academic, New York, 1960).

${ }^{28}$ P. Hirsch, A. Howie, R. B. Nicholson, D. W. Pashley, and M. J. Whelan, Electron Microscopy of Thin Crystals (Krieger, Malabar, FL, 1977).

${ }^{29}$ T. Kuzuba, K. Era, T. Ishii, and T. Sato, Solid State Commun. 25, 863 (1978).

${ }^{30}$ R. Geick, C. H. Perry, and G. Rupprecht, Phys. Rev. 146, 543 (1966).

${ }^{31}$ R. J. Nemanich, S. A. Solin, and R. M. Martin, Phys. Rev. B 23, 6348 (1981).

${ }^{32} \mathrm{~S}$. Tanuma and K. Okabe (private communication); Extended Abstract of the Symposium on Graphite Intercalation Compounds, edited by M. S. Dresselhaus, G. Dresselhaus, and S. A. Solin (Materials Research Society, Pittsburgh, PA, 1986), p. 196. 